\title{
Evolution of the turbulent/non-turbulent interface of an axisymmetric turbulent jet
}

\author{
M. Khashehchi ${ }^{1}$ - A. Ooi $^{2}$ • J. Soria ${ }^{3}$ - I. Marusic ${ }^{2}$
}

Received: date / Accepted: date

\begin{abstract}
Measurements of a turbulent round air jet, using Particle Image Velocimetry (PIV), were made to investigate the dynamics and transport processes at the continuous bounding interface between the turbulent and non-turbulent regions $(\mathrm{T} / \mathrm{NT})$ of the jet flow. The Reynolds number of the jet was in the range $R e_{d}=3000$ to 6500 based on jet diameter with measurements taken between 0 and 40 jet diameters from the jet nozzle exit, which covers the developing and self-similar region of the jet. A velocity thresholding technique was used to define the $\mathrm{T} / \mathrm{NT}$ interface. The results based on this methodology were found to agree well with previously published results (Westerweel et al. [2005, 2009] and Anand and Boersma [2009]). The evolution of the turbulence characteristics at the interface was investigated using conditionally averaged axial velocity, azimuthal vorticity, turbulent intensity and Reynolds shear stress. A distinct change in behavior is observed between the near-field region, $0 \leq x / d \leq 8$, and the far field, self-similar region, $x / d>20$. Profiles of the conditional mean axial velocity demonstrates no clear jump in the conditional mean axial velocity at the interface for $x / d<8$. However, the emergence of
\end{abstract}

\section{Khashehchi}

E-mail: m.khashehchi@uq.edu.au

School of Mechanical and Mining Engineering, The University of Queensland, QLD 4072, Australia:

A. Ooi · I. Marusic

Department of Mechanical Engineering, University of Melbourne, Victoria, 3010, AUSTRALIA

J. Soria

Laboratory for Turbulence Research in Aerospace and Combustion, Department of Mechanical and Aerospace Engineering, Monash University, VIC 3800, Australia Department Of Aeronautical Engineering, King AbdulAziz University, Jeddah, Kingdom of Saudy Arabia the jump is observed at $x / d \approx 8$ and the magnitude of the jump continues to increase between $8<x / d<20$ before reaching a steady value in the self-similar region, $x / d>20$. All the scaled statistics show self-similar behavior downstream of $x / d>20$. Similar behavior is observed for the peak in the azimuthal vorticity and the jump in the Reynolds shear stress profiles. The evolution of the rate of entrainment also confirms the creation and growth of the jump in the mean conditional velocity profiles in the developing region of the jet.

Keywords PIV $\cdot \mathrm{T} / \mathrm{NT}$ interface $\cdot$ developing area turbulent round jet

\section{Introduction}

The focus of this study is on the interface between the non-turbulent and turbulent regions in shear flows, where turbulent entrainment takes place (Hinze [1975], Tsinober [2001] and Hunt et al. [2001]). Specifically, the interest here is the turbulent round jet, where the size of this thin region is between one-two orders of magnitude smaller than the integral length scale of the flow (Anand and Boersma [2009], Bisset et al. [2002] and Hunt et al. [2006]). The most important feature of this region is the continuous exchange that occurs locally at the interface that is essential for the transport of heat, mass and momentum between the irrotational surrounding region and the fully-turbulent region of the jet. The physical mechanism of the entrainment process is not yet completely understood. Considerable effort has been invested in this field to understand the evolution and dynamics of the flow structure in the proximity of the interface. It is well known that the vorticity layer leaving the nozzle becomes unstable, forming Kelvin-Helmholtz waves and then forming vortex 
rings that convect downstream. These organized vortex rings eventually breaks down into more complex structures within a few diameters of the jet nozzle (see white square in figure 1). The mechanism of entrainment in the jet far-field can be viewed as a stochastic process, which is governed by either small-scale viscous structures, large-scale structures or both mechanisms. As recently discussed by Philip and Marusic [2012], historically, two different physical models have been proposed to describe the entrainment mechanism. One model due to Corrsin and Kistler [1954], suggests that entrainment is associated with small scale ( "nibbling") eddy motions and this suggestion is supported by a number of experimental and numerical studies (see Westerweel et al. [2005], Mathew and Basu [2002], Da-Silva and Pereira [2008], Holzner et al. [2007] and Holzner et al. [2008]).

However, quantitative measurements (e.g., Kovasznay et al. [1970]) failed to confirm the existence of this interfacial layer. Brown and Roshko [1974] showed that the former classical picture of jet entrainment may not be correct and that transport on large-scales ( "engulfing") may be the dominant process for the entrainment of non-turbulent flow particles toward the turbulent region. In the Brown and Roshko [1974] study, they visualized mixing layers using spark shadow photography which were seen to be dominated by large coherent structures. High-speed movies also showed that these structures convect at nearly constant speed, and increase their size and spacing discontinuously by amalgamation with neighboring structures, creating large-scale structures in the jet far-field. According to this description of the jet flow, the turbulent entrainment process is dominated by large scale flow structures ( "engulfing"), where non-turbulent regions are pushed into turbulent regions. This "engulfing" process is supported by various experimental studies (Dahm and Dimotakis [1987], Dimotakis [2000], Mungal et al. [1991] and Ferre et al. [1990]).

Westerweel et al. [2002] obtained experimental support for "nibbling" from a combination of PIV and PLIF (planar laser induced fluorescence) on an axisymmetric free jet. This yielded a large set of data including information on the instantaneous location of the interface between the turbulent and non-turbulent regions. A threshold on the concentration value of dye was used to detect the interface location. The resulting profile of the mean conditional axial velocity showed a distinct jump at the $\mathrm{T} / \mathrm{NT}$ interface which agreed with the DNS based results of Bisset et al. [2002]. A further study by Westerweel et al. [2005] of the mechanics and transport processes at the interface also showed the existence of a finite jump in the conditional mean axial velocity at the interface, as well as a small peak in the vorticity profile

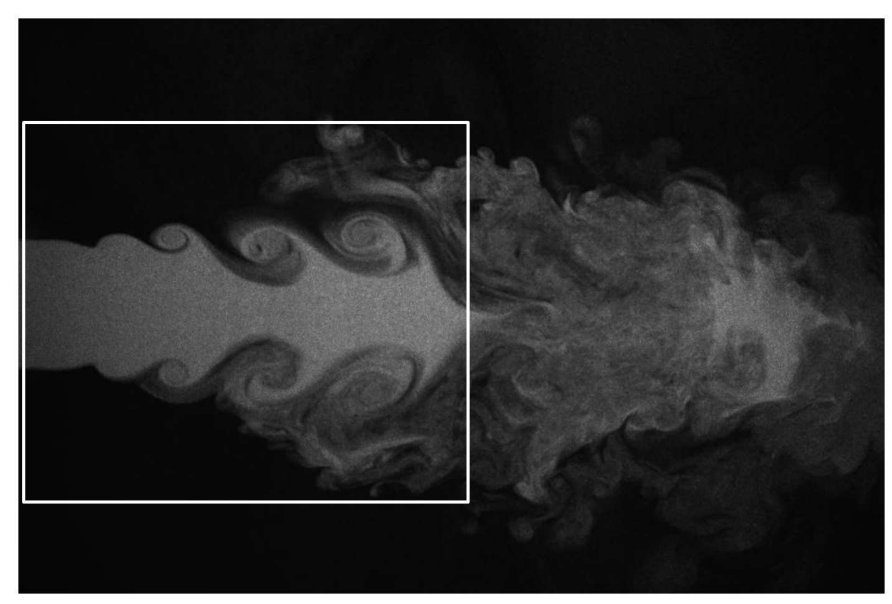

Fig. 1 Instantaneous particle seeded image of Jet2 experiment. In this figure only the jet was seeded for visualization purposes.

at the T/NT interface. They found that the magnitude of the vorticity peak is proportional to a jump in the axial velocity profile, which agrees with the results of Holzner et al. [2006]. In a more recent study, Westerweel et al. [2009] showed that the mean outward boundary interface velocity, $E_{b}$, is proportional to a fraction of the value of the jump in the mean axial velocity and Reynolds shear stress value where this ratio is found to be 0.07 of the mean centerline velocity.

To date, the experimental and computational investigations of the $\mathrm{T} / \mathrm{NT}$ interface are predominantly based on measurements in the self-similar region. The region upstream of the self-similar region has received little attention. The present study aims to provide more information by investigating the $\mathrm{T} / \mathrm{NT}$ interface of a turbulent jet upstream of the self-similar jet up to and including the self-similar region. Of particular interest is the emergence and axial development of the jump in mean conditional axial velocity, from the jet exit to the self-similar region. Thus, the characteristics of the interface in a transitional turbulent round jet are investigated in detail. Therefore, the first objective of this study is to examine the spatial evolution of the interface characteristics in the region up to $30 d$ downstream of the jet nozzle, where $d$ is the jet diameter.

In order to observe the transitional process of the flow, results of high resolution 2D-2C Planar-PIV of the Jet1 (facility of the LTRAC laboratory located at Monash University) at $R e_{d}=3000$ were initially used. In addition the Planar-PIV results of Jet2 (facility of Walter Bassett Aerodynamic Laboratory lab located at the University of Melbourne) at twice the Reynolds number of the first experiments were also performed in the near field region of the round jet. This was done to 
validate the statistical results of the first experiment in this region with sufficient and improved spatial resolution than was possible with the Jet1. The quality and validity of the experimental measurements were established by comparison with other published results in the self-similar region. One of the novel aspects of the present study is the use of two large sensors simultaneously $(P C O-4000 \mathrm{CCD}$ arrays with $4008 \times 2670$ pixels resolution), providing high spatial resolution PIV measurements. Table 1 summarizes the details of several previous studies compared with the present study.

\section{$2 T / N T$ Interface Detection Methodology}

In this study, a thresholding method is used to detect the T/NT interface. Since the measurements in the present study are limited to two components of velocity $(U, V)$, we will use the velocity criteria first proposed by Holzner et al. [2006]. In this algorithm, a flow field is defined to be in a turbulent region when

$$
\frac{U}{U_{o}} \geq k
$$

where $k$ is a threshold value and $U_{o}$ is the axial velocity at the nozzle exit. Conversely, the flow is considered to be irrotational whenever $\frac{U}{U_{o}}<k$. Here we use a threshold value of $k=0.03$, as was used by Anand and Boersma [2009], who showed that the detected interface position is insensitive to small changes in the value of $k$. A sample of this interface detection method with contours of the instantaneous vorticity field is shown in figure 2. It is clear that this interface detection algorithm encompasses most of the vorticity field and at least in a visual sense distinguishes between the turbulent and non-turbulent regions.

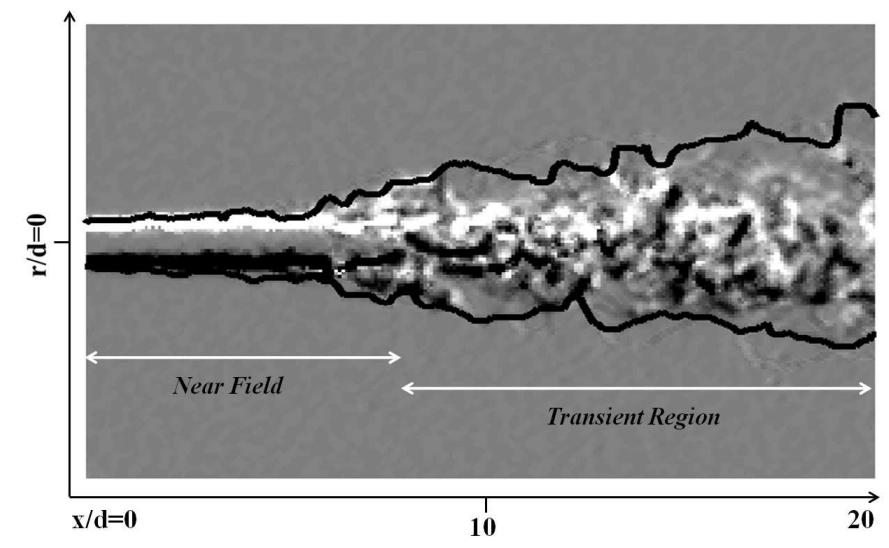

Fig. 2 Contours of vorticity $\Omega_{z}$ and the jet interface (colors are normalized between 1 and -1 ).
The following process was undertaken in order to measure mean conditional values and profiles for each flow property. Firstly, the value of $\frac{U}{U_{o}}$ is computed for all data points in the instantaneous flow field and the location of the interface is defined where $\frac{U}{U_{o}} \approx 0.03$. Then a new origin of coordinates at the location of the interface, $O^{\prime}\left(x, r-r_{i}\right)$, is defined, as indicated in figure 3. Finally, temporal averages of all the data over the entire samples with respect to the location of the new origin are computed to obtain the conditional average of the flow property.

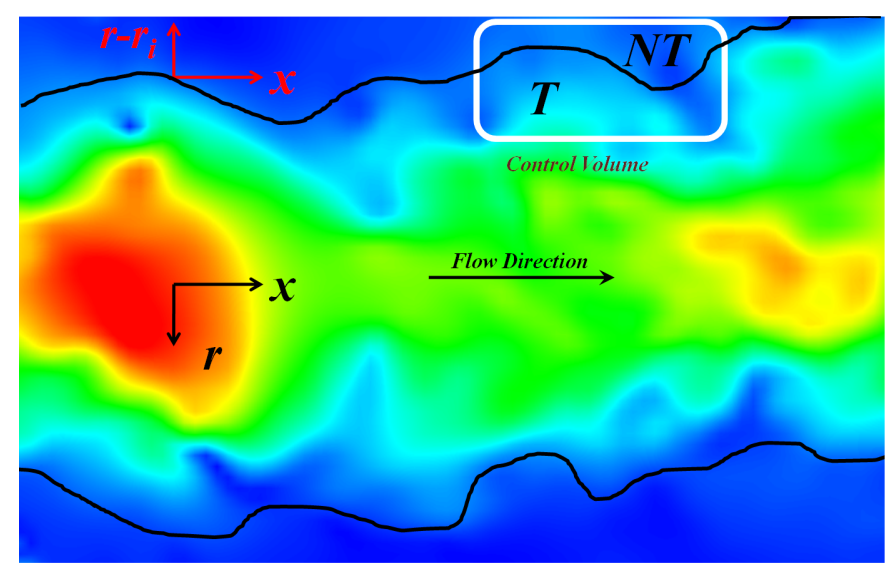

Fig. 3 Instantaneous velocity field in the transitional region of a round turbulent jet. The $\mathrm{T} / \mathrm{NT}$ interface is shown.

The study of the flow characteristics in a cross section of the interface can be achieved by analyzing a control volume on that area including the non-turbulent, interface and turbulent regions (see figure 3). Following Westerweel et al. [2009], if we assume $U$ as a property of the flow, a simple control volume analysis yields:

$E_{b} \Delta U=-F_{U}$,

where $F_{U}$ is the flux of $U$ into the control volume and $E_{b}$ is the velocity of interface toward the irrotational region and normal to the interface curve (figure 3). Holzner and Luthi [2011] showed that the shape of the boundary is continuously deformed to maintain the volume flux value as the jet expands. Equation 2 suggests a nonzero value for $\Delta U$ for flux of $U$ to exist (see e.g. figure 2 of Westerweel et al. [2009]). Westerweel et al. [2009] showed that:

$E_{b} \approx \frac{\Delta(u v)}{\Delta U}$

where the ratio of the jump in the mean conditional axial and Reynolds shear stress profiles is proportional to the velocity of the interface. The magnitude of the 
Table 1 Experimental parameters.

\begin{tabular}{|l|c|c|c|c|c|}
\hline \hline Parameter & Jet1 & Jet2 & Westerweel [2005] & Westerweel [2009] & Agrawal [2002] \\
\hline Nozzle Diameter $(\mathrm{mm})$ & 2 & 25 & 1 & 1 & 2 \\
Measurement & $0<x / d<40$ & $0<x / d<6$ & $60<x / d<100$ & $60<x / d<100$ & $110<x / d<175$ \\
Flow Type & Air & Air & Water & Water & Water \\
$R e_{d}$ & 3000 & 6500 & 2000 & 2000 & 3000 \\
Sensor Size & $2 \times 4008 \times 2670$ & $4008 \times 2670$ & $992 \times 1004$ & $992 \times 1004$ & $1026 \times 1000$ \\
Magnification & 0.93 & 0.35 & 0.27 & 0.27 & 0.07 \\
Resolution/diameter & 0.144 & 0.0328 & 0.7 & 0.37 & 1 \\
\hline
\end{tabular}

ratio of these two properties (and hence $E_{b}$ ) in the selfsimilar region of the jet has been measured and found to be $E_{b}=\Delta(u v) / \Delta U \approx 0.07 U_{c}$.

\section{Experimental Setup}

A brief summary of the experimental techniques is included in this section. Figure 4 shows a schematic of the experimental setup used for the Jet1 experiments. The facility consists of an axi-symmetric air jet with a nozzle diameter of $2 \mathrm{~mm}$. High pressure air was provided by the Monash University compressed air supply and passed through a large high pressure vessel before entering the settling chamber of the jet. The air flow then enters a $19: 1$ axisymmetric contraction before issuing from the circular jet nozzle. The mean velocity of the air at the jet outlet was $23 \mathrm{~m} / \mathrm{s}$ resulting in a Reynolds number based on the jet exit diameter of 3000 . The Jet2 apparatus was similar to the Jet1 except that the exit diameter of the nozzle was $25 \mathrm{~mm}$ with a mean jet velocity of $4 \mathrm{~m} / \mathrm{s}$ resulting in a Reynolds number equal to 6500 .

Single-exposed image cross-correlation PIV measurements were used to measure both the rotational turbulent flow of the jet and the irrotational ambient flow outside the jet. In order to ensure good measurements in both the turbulent jet and non-turbulent ambient regions two seeders were operated during the PIV experiments. The compressed air flow for the jet was seeded in the high pressure vessel, which was developed inhouse and contained eight ultrasound devices (APC Nebuliser) that generated nominally $2 \mu \mathrm{m}$ diameter water particles. The external ambient air was seeded with a fog-generator that also produced nominally $2 \mu \mathrm{m}$ diameter water particles. Since the latter seeding process generated a small movement in the surrounding environment of the jet, each set of experiments was carried out two minutes after the ambient air was seeded to minimise disturbances of the ambient air by external factors.

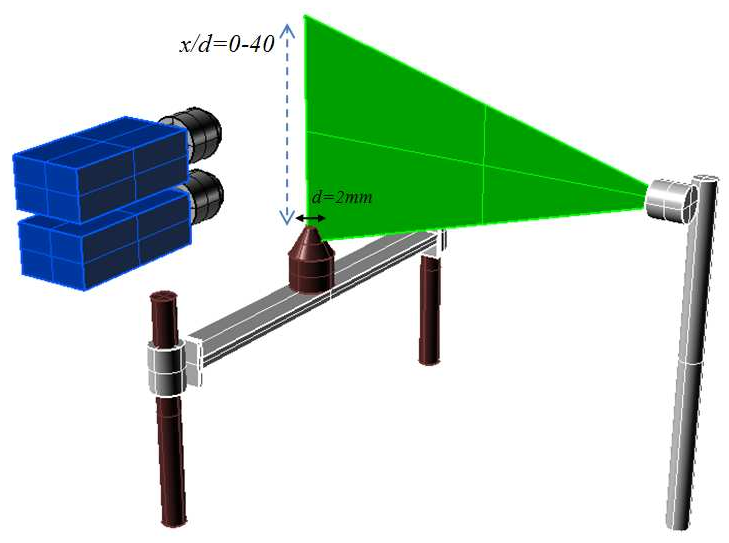

Fig. 4 Schematic of the experimental configuration.

The seed particles were illuminated during the PIV experiments by a Big Sky Nd-YAG laser capable of a maximum $180 \mathrm{~mJ}$ per $5 \mathrm{~ns}$ pulse at $532 \mathrm{~nm}$ wavelength. The laser sheet was formed using a set of spherical and cylindrical lenses, resulting in a light sheet thickness of $2 \mathrm{~mm}$. The laser illuminated a plane perpendicular to the cameras along the jet axis. Measurements along a large domain in the jet flow direction with high spatial resolution were enabled by employing two PCO4000 cameras $(4008 \times 2700$ pixels $)$ with $200 \mathrm{~mm}$ Micro Nikkor lenses set at an f-stop number of 4 resulting in a magnification of 0.93 with a corresponding field of view for each camera of $40 \times 26 \mathrm{~mm}$ for the smaller diameter jet experiments. For the larger jet diameter experiments $105 \mathrm{~mm}$ Sigma lenses were used with a magnification of 0.35 . The single-exposed double-frame acquisition frequency was $2 \mathrm{~Hz}$. 2000 statistically independent velocity samples were acquired. 


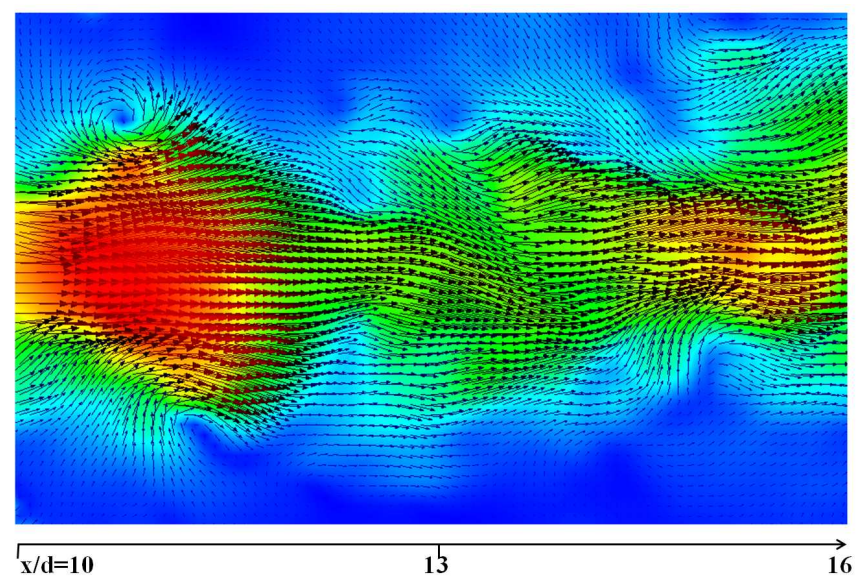

Fig. 5 The instantaneous velocity field in the transitional region of the turbulent round jet (Jet2).

The single exposed image pairs were analysed using the multigrid cross-correlation digital PIV (MCCDPIV) algorithm described in Soria et al. [1999]. Table 2 indicates the interrogation parameters used in the analysis of the PIV images. The estimated depth of field (dof) was calculated to be 20 pixels with the current camera setup. Details of the performance, accuracy and uncertainty of the MCCDPIV algorithm with applications to the analysis of single exposed PIV (2C-2D) and holographic PIV (HPIV) (3C-2D) images have been reported in Soria [1998] and von Ellenrieder et al. [2001], respectively. The uncertainty relative to the maximum velocity in the velocity components at the $95 \%$ confidence level for these measurements is $0.3 \%$.

Table 2 PIV image acquisition and analysis parameters

\begin{tabular}{cc}
\hline \hline Parameter & Quantity \\
\hline$\delta t$ Jet1 & $7 \mu \mathrm{s}$ \\
$\delta t$ Jet2 & $65 \mu \mathrm{s}$ \\
Grid Spacing & 16 pixels \\
$I W_{0}$ & 32 pixels \\
$I W_{1}$ & 64 pixels \\
dof & 20 pixels \\
\hline
\end{tabular}

The azimuthal vorticity, $\Omega_{z}$, was also calculated from the MCCDPIV velocity field measurements using a local least-squares fit procedure to the velocity field. A sample of the instantaneous velocity field from the PIV data in the turbulent region of the jet is shown in figure 5 .

\section{Results and Discussions}

\subsection{Near-Field Region}

The statistics of the velocity field of the Jet2 in the near-field region are presented first. These results are used to confirm the characteristics of the interface of the smaller diameter jet in the near-field region as the Jet2 results have significantly high spatial resolution in the near-field region. All the statistics are normalized with the mean local centerline velocity, $U_{c}$, and jet half width $\delta_{1 / 2}$. Figure 6 shows the mean axial velocity and Reynolds shear stress profiles for several near-field locations. The profiles are symmetric within the PIV spatial resolution and show the evolution of the statistics from $x / d=0$ to 6 . Assuming that the jet exit velocity has a top-hat distribution, the mean centerline velocity $U$ is constant along the potential core.

4.2 Evolution of the One- and Two-Point Statistics in the Transitional Region

Hereafter only the results of the small diameter jet (Jet1) in the transitional region will be presented and some of the graphs, especially in the near-field region, will be compared with the results of the large diameter jet (Jet2) experiment. The results for the mean centreline velocity $U_{c}$ and the jet half-widths $\delta_{1 / 2}$, based on the velocity field, are presented in figure 7 . As expected for a near-field turbulent round jet, $U_{c}$ has a constant value. The jet half-widths $\delta_{1 / 2}$ increase linearly proportional to the distance from the nozzle. It should be noted that the rate at which the jet half-width grows and the rate at which the jet centerline velocity decays originates from different virtual origins as indicated in figure $7 \mathrm{~b}$, where the virtual origin of the mean centerline velocity and the jet half-width are located at $x / d=3$ and 2 , respectively.The influence of the downstream distance on the integral length scales in the jets can be considered by measuring integral length scale $L$ (equation 4 ) as a function of $x / d$,

$L=\int_{0}^{\infty} \frac{\langle u(x) \times u(x+r)\rangle}{\left\langle u(x)^{2}\right\rangle^{0.5}\left\langle u(x+r)^{2}\right\rangle^{0.5}} d r$.

Figure $7 \mathrm{c}$ displays the centreline evolution of the integral scale for Jet1. It can be observed that the integral scale consistently increases as the flow evolves toward its self similar state.

The axial mean velocities, normalized by the centerline velocity, $U_{c}$, at different axial positions $x / d$, are presented in figure 8 . The top-hat profile at the nozzle exit decays as $x / d$ increases, reaching a self-similar profile at $x / d \approx 15$. This result agrees with the data of 


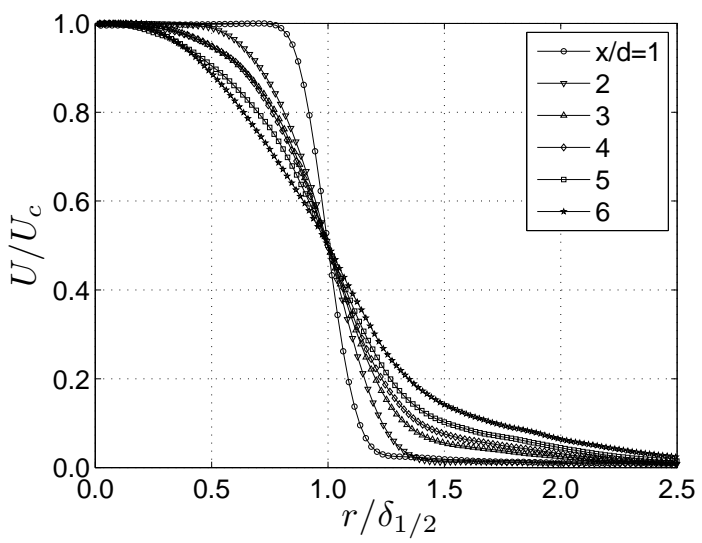

(a)

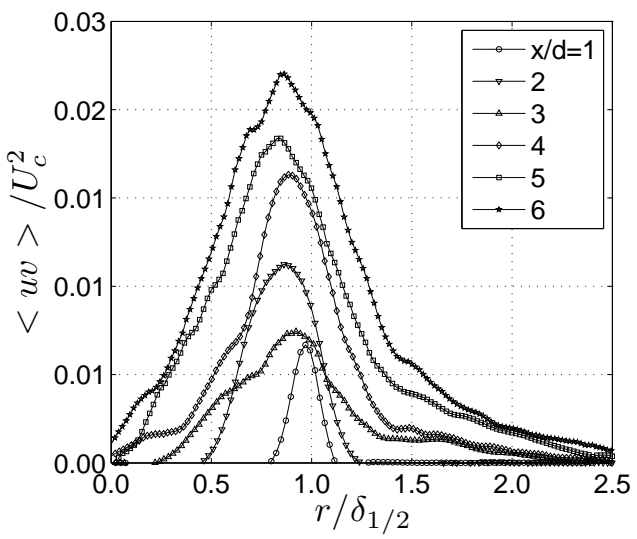

(b)

Fig. 6 Profiles of a) mean axial centerline velocity, and b) Reynolds shear stress, for several different distances from the jet exit of Jet2 experiment.

Weisgraber and Leipman [1998] in the transitional region and Hussain [1998] and Panchapakesan and Lumley [1993] obtained in the self-similar region. The corresponding axial and radial turbulence intensity profiles, for $x / d<20$ are displayed in figures 9 and 10 , respectively. The axial and radial turbulence intensities have nearly the same shape. In general, the axial turbulence intensity, $u$, is greater than the radial turbulence intensity, $v$, along the axial direction. At $x / d=15$, the turbulence intensities are clearly anisotropic and the reason for this is likely due to turbulence structure influences, which will be revisited below. One of the most interesting features of the transition region is the way in which the turbulence intensities re-distribute across the jet as the flow evolves downstream. That said, at $x / d=15$, the time averaged turbulence distributions are remarkably similar in shape. Figure 11 presents the Reynolds shear stress, normalized by the square of the centerline velocity $U_{c}^{2}$. The maximum Reynolds stress moves toward the jet centerline with downstream distance.

The Taylor micro-scale $\lambda_{T}$ can be estimated from the axial $\mathrm{rms}$ velocity and axial velocity gradient using the following equation:

$\lambda_{T}=\frac{\sqrt{\overline{u^{2}}}}{\sqrt{\frac{\partial u^{2}}{\partial x}}}$

Using this equation and with $\sqrt{\overline{u^{2}}} \approx 0.27 U_{c}$ (figure 9 ), it is found that $\lambda_{T}$ is approximately $0.9 \mathrm{~mm}$, which is nominally three times larger than the equivalent dimension of the interrogation domain. Using the estimated value of rate of dissipation, $\varepsilon=15 \nu \frac{\overline{u^{2}}}{\lambda_{T}^{2}}$, an estimation for the Kolmogorov length scale $\eta=\left(\frac{\nu^{3}}{\varepsilon}\right)^{\frac{1}{4}}$ is found to be $75 \mu \mathrm{m}$. Therefore, the spatial resolution of the PIV data $(0.28 \mathrm{~mm})$ is 3.8 times the Kolmogorov scale. Moreover, at the self-similar regime, the Reynolds number based on the Taylor micro-scale and on the root mean square of the streamwise velocity is equal to $R e_{\lambda} \sim 122$.

\subsection{Mean Conditional Statistics}

Results from the self-similar region will be presented first. The results of mean conditional average (data conditioned on the location of the interface) of the axial velocity, azimuthal vorticity and Reynolds shear stress in the self-similar region $(x / d=25)$ of the jet, scaled with the half-jet width, $\delta_{1 / 2}$, and normalized by the mean centerline velocity, $U_{c}$, will now be considered. Figure 12 shows several instantaneous and temporal averaged conditional profiles of the axial velocity. The overbar in these graphs indicates temporal conditional averages. It is clear from this graph that none of the instantaneous profiles shows a jump at the interface. However, there is clearly a jump in the mean temporal conditional average, $\bar{U}$, at the interface. This is consistent with the data presented by other investigators (Westerweel et al. [2005, 2009]).

Conditional mean axial velocity profiles are shown in figure 13a. A created small jump $(\Delta U)$ in the mean conditional velocity distribution is observed in the figure 13a. Westerweel et al. [2009] measured the size of this jump using two mathematical models with and without jumps at the interface (figure 13a inset) and found the mean axial jump at the interface to be $0.09 U_{c}$. Figure 13b shows that the value of $\overline{u v}$ is not equal to zero at the interface. Note that this nonzero property in the irrotational side does not contribute to the tur- 


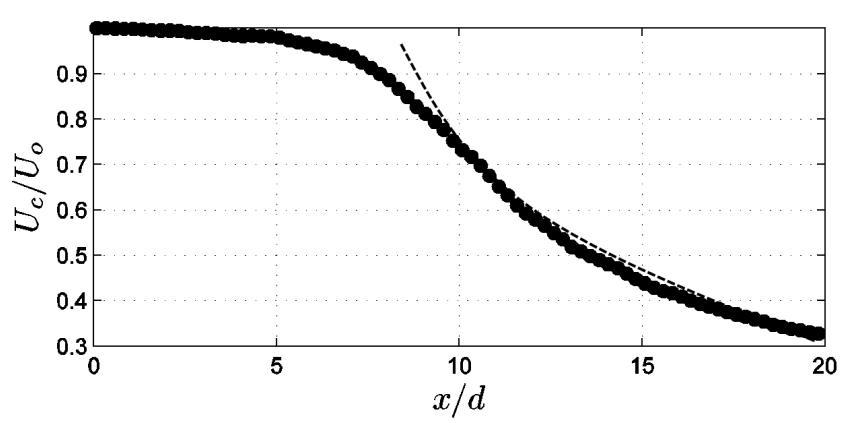

(a)

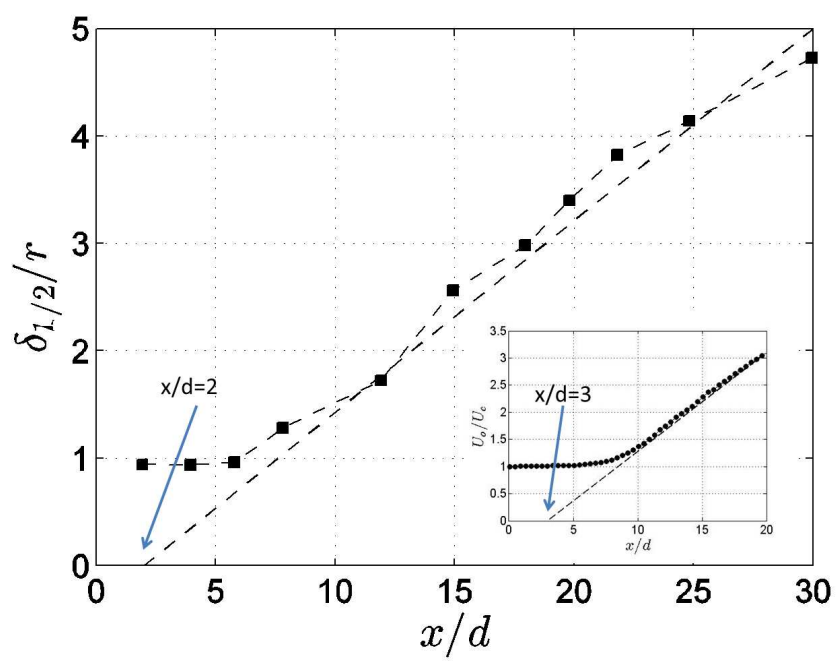

(b)

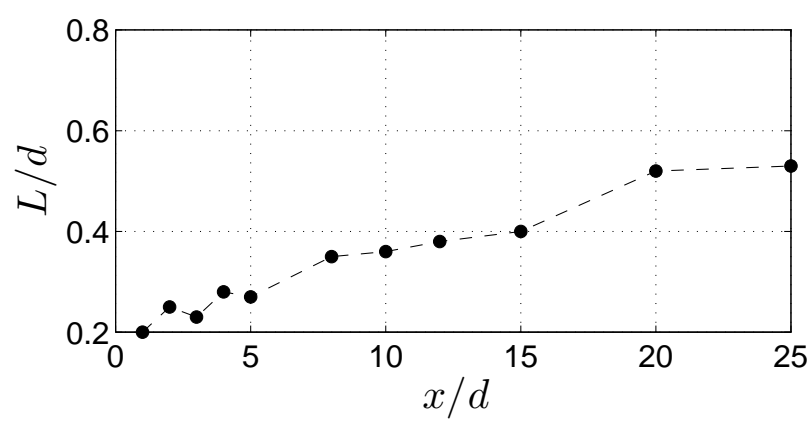

(c)

Fig. 7 Mean centreline velocity (a), jet half-width profiles as a function of distance from the jet exit (b) and the evolution of the integral length scale, $L$ (c). Note that the inset of (b) shows the $U_{0} / U_{c}$, illustrating the virtual origin of the decay of jet centreline velocity.

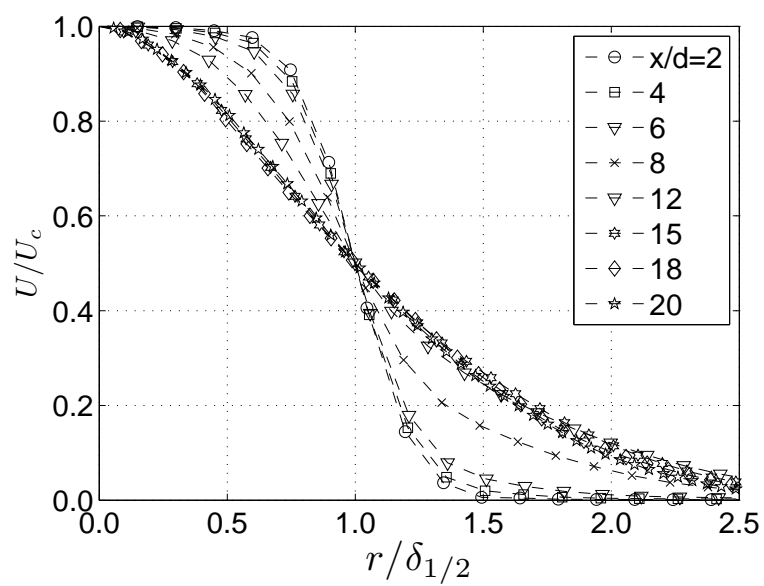

Fig. 8 Profiles of axial mean velocity at different axial positions.

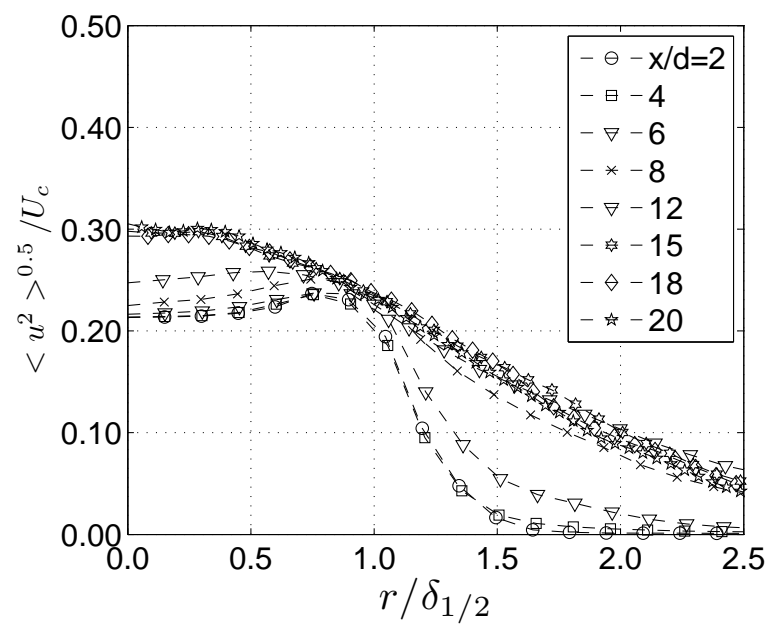

Fig. 9 Profiles of axial turbulence intensity at different axial positions.

bulent momentum transport (Pope [2000]). The magnitude of $\overline{u v}$ at the interface location is equal to 0.0037 which is in agreement with the finding of Westerweel et al. [2009].

Figure $13 \mathrm{c}$ shows the mean conditional azimuthal vorticity and it is seen that the height of the peak $\overline{\Omega_{z}}$ from our data is a bit higher than published data in the open literature. This may be caused by the choice of different interface detection algorithms and spatial resolution differences in the experimental data (Anand and Boersma [2009]). Nevertheless, the reason for this peak is the tendency of the vortex sheet structures to form at the border of the jet (Westerweel et al. [2009]). Figure $13 \mathrm{c}$ also shows a zero value $\overline{\Omega_{z}}$ in the irrotational 


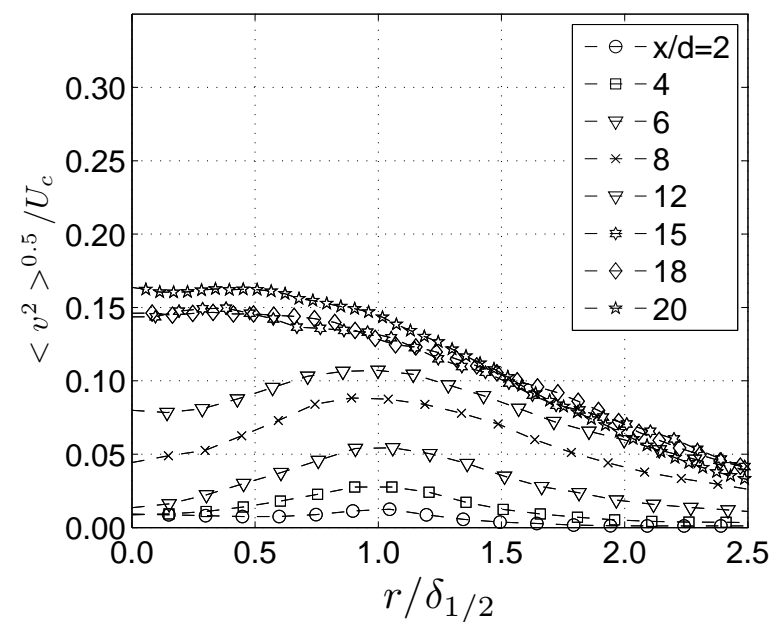

Fig. 10 Profiles of radial turbulence intensity at different axial positions.

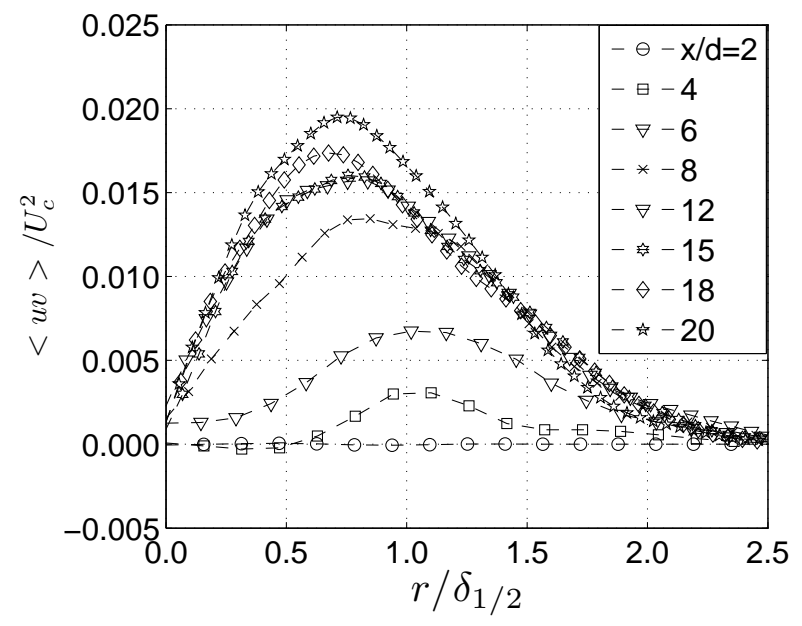

Fig. 11 Profiles of Reynolds shear stress at different axial positions.

region, followed by a more or less constant value for $\overline{\Omega_{z}} \delta_{1 / 2} / U_{c}$ in the turbulent side at $\left(r-r_{i}\right) / \delta_{1 / 2} \approx 0.3$.

One important question in the context of the interface is the thickness of the viscous superlayer which can be seen as the inner layer of the viscous superlayer (Westerweel et al. [2005, 2009]). As seen in figure 13 , substantial changes in the momentum and vorticity magnitude appear in this thin region, therefore it is now valuable to measure the thickness of this layer and compare with the resolution of the PIV results. The thickness of the T/NT interface can be defined by the width of the peak in the mean conditional azimuthal vorticity profile. Note that in the present study the width of this peak is limited to the resolution of the measurements. Figure 14 again shows the conditional mean azimuthal

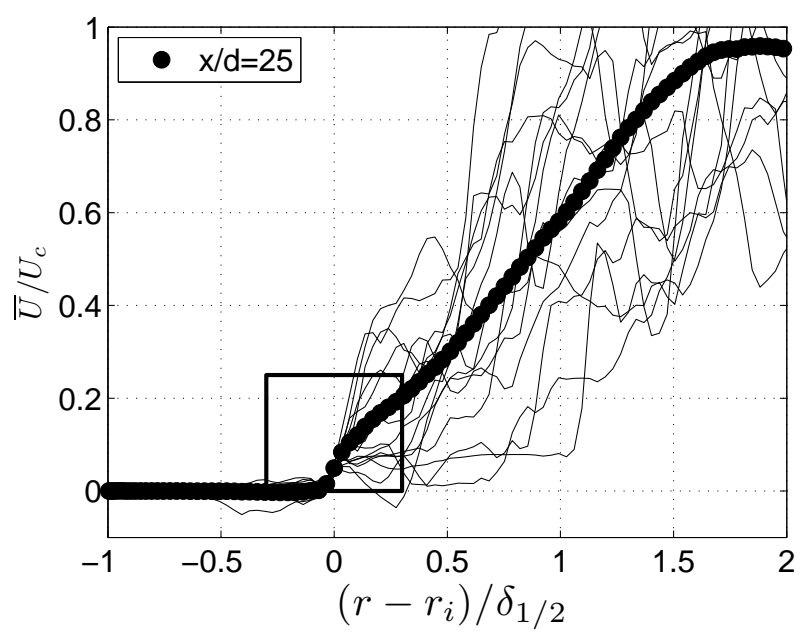

Fig. 12 Temporal-average conditional axial velocity with some instantaneous samples. The gray lines are some sample profiles.

vorticity profile where the horizontal axis is normalized with the local Taylor micro-scale and Kolmogorov length scale. The distance between the point at which the peak starts to appear and the maximum vorticity peak can be defined as half of the interface thickness. Thus, the thickness of the interface for the present study is approximately to the Taylor micro-scale, which is the same value observed in the experimental data of Westerweel et al. [2005] and in the DNS study of a plane jet by da Silva and Taveira [2010].

In the subsequent section, data showing the evolution of mean conditional axial velocity, azimuthal vorticity and Reynolds shear stress will be presented and compared with the self-similar turbulent region in order to shed light into the development interface structure of turbulent round jets.

\subsection{Evolution of Conditional Statistics}

In this section, we will apply the ideas and analysis introduced in the last section to the data obtained in the near field, transient and fully turbulent regions of the turbulent jet in order to study the evolution characteristics of the jet interface.

Figure 15 shows two instantaneous detected interfaces superimposed with the vorticity field. Transition is seen to occur somewhere between $5<x / d<10$. The starting point of transition, however, can be defined as the end of the potential core where the mean centerline velocity profile falls from its maximum (figure 7a). A better definition of the starting point of transient region can be achieved by looking at the characteristics 


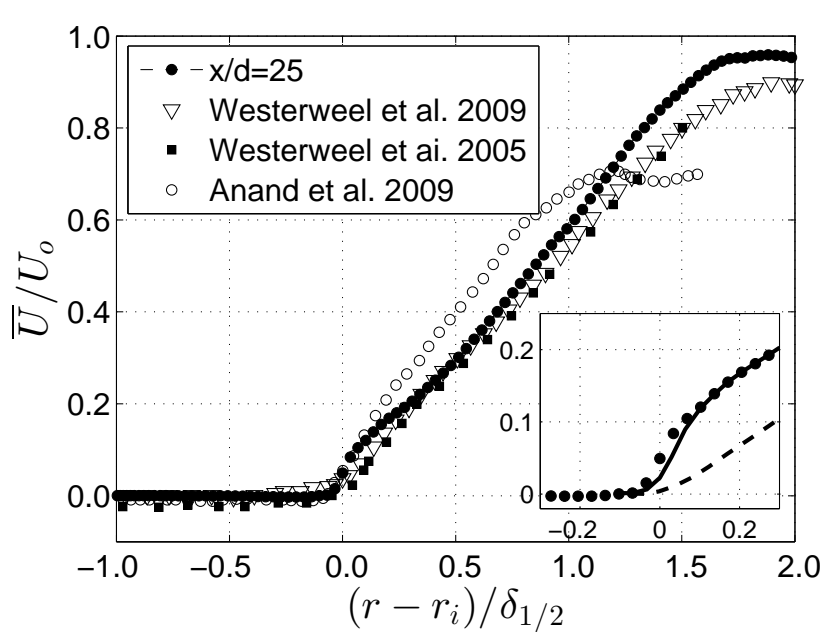

(a)

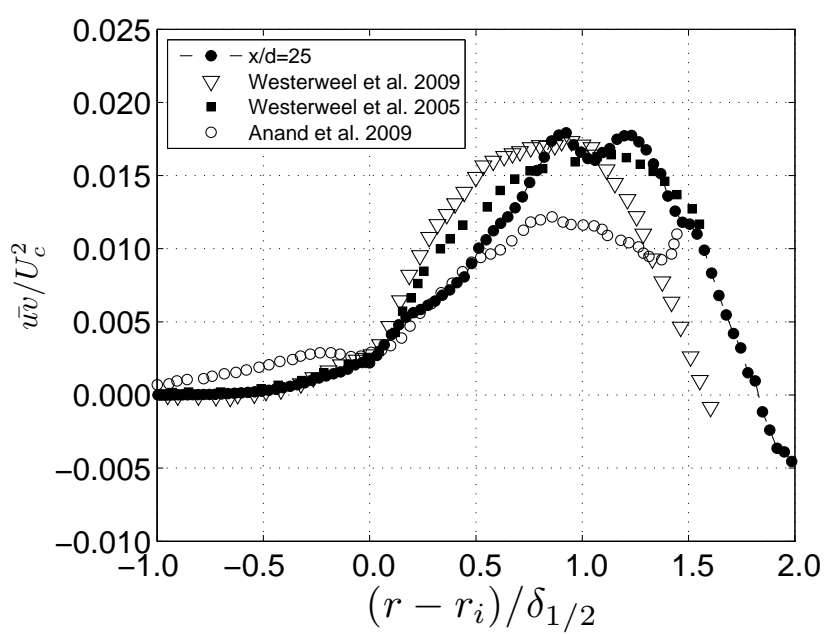

(b)

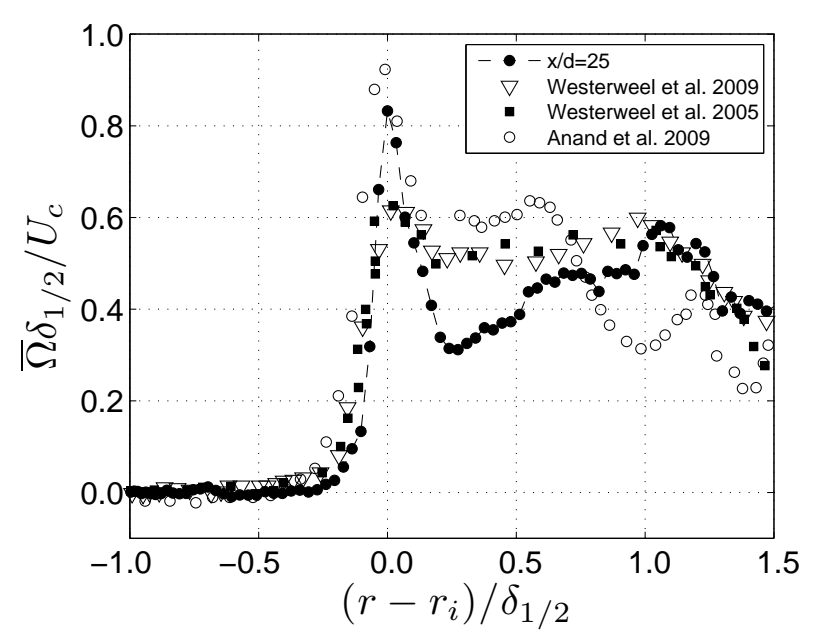

(c)

Fig. 13 Mean conditional average of the axial velocity, Reynolds shear stress and azimuthal vorticity with respect to the interface location $\left(r-r_{i}\right)$ in self-similar region $(x / d=25)$ of the jet.

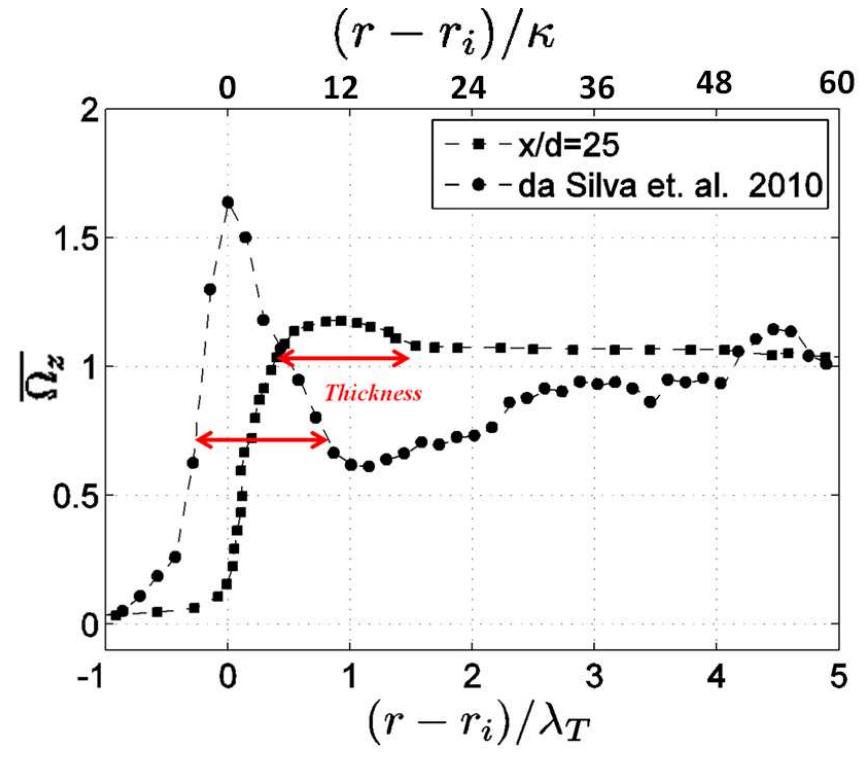

Fig. 14 Profile of mean conditional azimuthal vorticity, horizontal axis is normalized with the local Kolmogorov length scale and the local Taylor micro-scale.

(a)

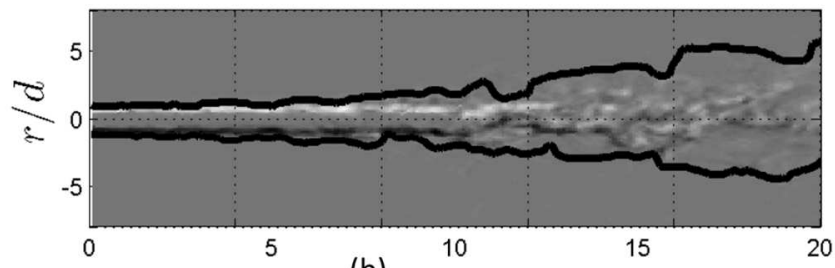

(b)

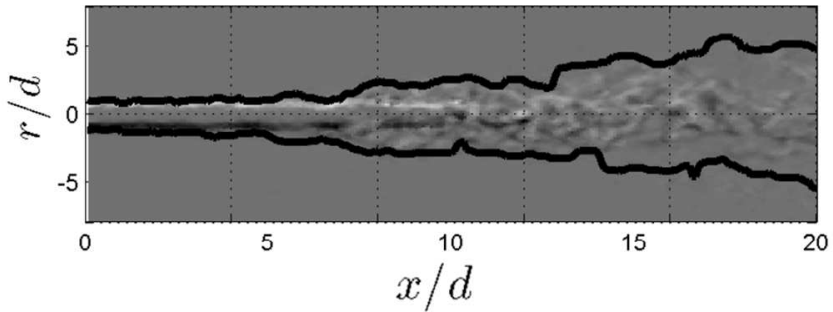

Fig. 15 Two instantaneous interface lines superimposed with the vorticity field $(a, b)$.

of the jet at the interface location in these particular locations. Conditional statistics are determined by averaging data at fixed distances relative to the envelope for both near-field and transition areas as well as the self-similar regions.

In figure 16, we present the mean conditional axial velocity profile for 8 (figure 16a) and 3 (figure 16b) different axial locations with respect to the jet exit. As shown in the graphs, $\bar{U}$ is equal to zero at the nonturbulent side of the interface and increases rapidly after passing the interface in the turbulent region. Another interesting observation is that there is no clear 


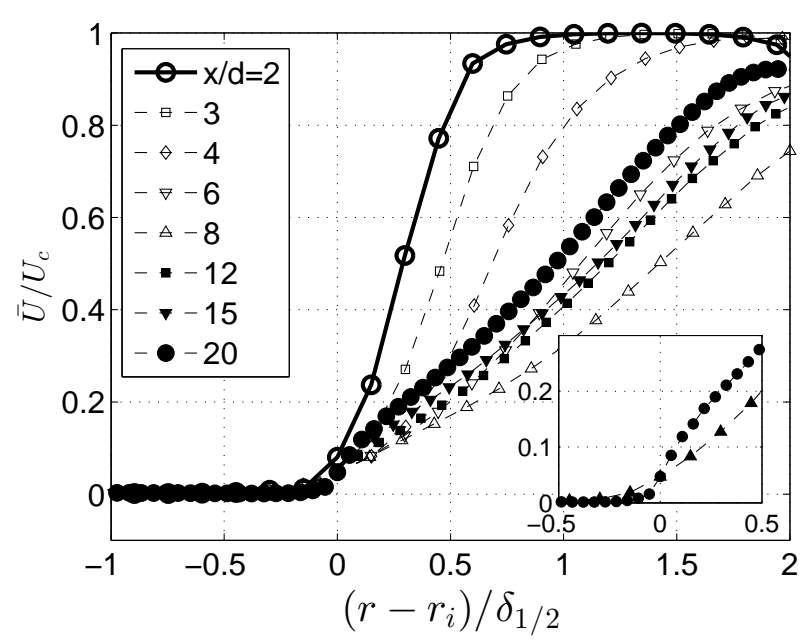

(a)

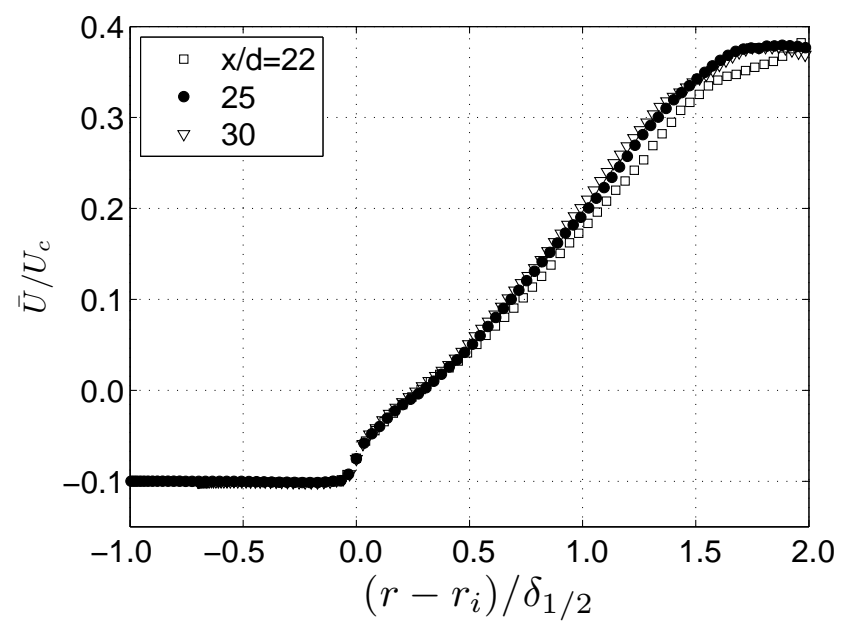

(b)

Fig. 16 Profiles of mean conditional axial velocity for several different distances from the jet exit, in the developing area (a) and the self-similar turbulent region (b) of the jet.

jump in the mean conditional profile of $\bar{U}$ in the graphs before $x / d=8$. For $0<x / d<8, \bar{U}$ appears to decay without any jump in the interface. To make a clear understanding of the jump at the interface, two graphs from the small box in the figure $16 \mathrm{a}(x / d=8$ and 20$)$ are selected and plotted as an inset inside figure 16a. No jump created in the near-field region may be caused by the limited spatial resolution in this region. In order to make sure that there is no jump in the mean axial velocity profile in this region a comparison was made with the results of the highly resolved Jet2 and the results are shown in figure 17.

At $x / d \approx 8$, a small jump in $\bar{U}$ begins to emerge, while for $8<x / d<20$, the magnitude of $\Delta U$ increases and the jump in $\bar{U}$ grows linearly. The flow in this re-

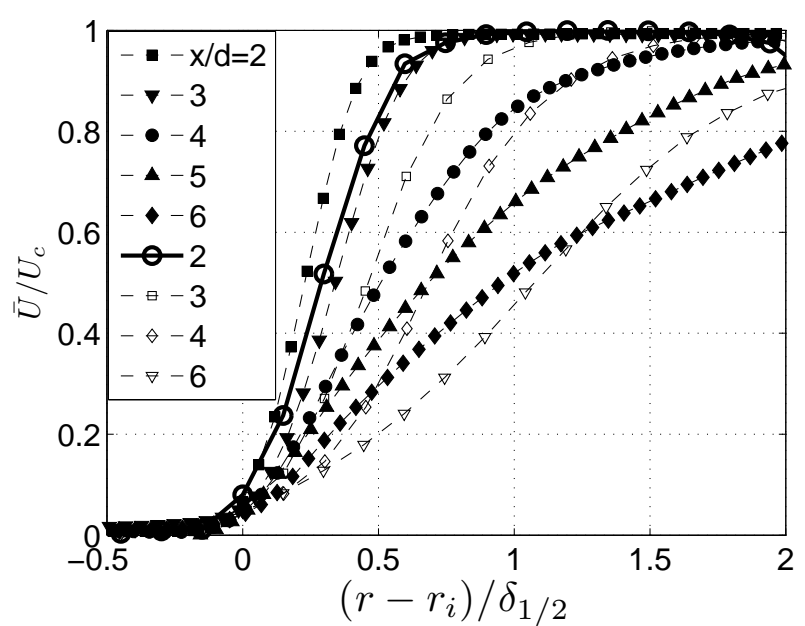

Fig. 17 Profiles of mean conditional axial velocity for several different distances from the jet exit. Solid markers are from the Jet2.

gion is quite unstable and the interface starts to fluctuate, caused by a small jump in $\bar{U}$. Finally, the profile assumes a self-similar shape for $x / d>20$ (figure $16 \mathrm{~b})$, consistent with published experimental results of Westerweel et al. [2005, 2009] and Anand and Boersma [2009]. The $\Delta U$ for different distances from the jet outlet is plotted in figure 18. The zero value of $\Delta U$ for $x / d<8$ is followed by a linear region, and reaches a plateau at around $0.09 U_{c}$ at $x / d=20$. Note that the measured $\Delta U$ in this experiment is limited to the finite spatial resolution of the PIV data.

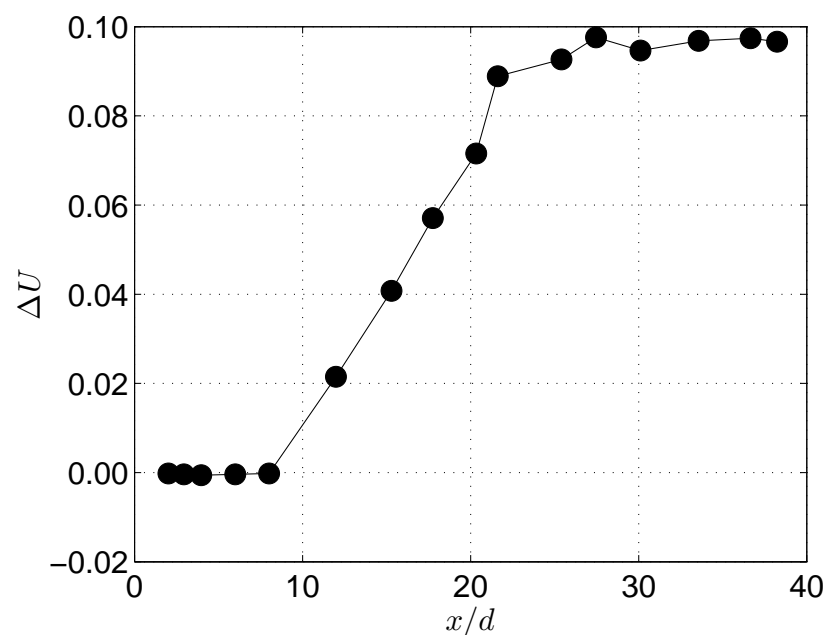

Fig. 18 The jump in the mean conditional axial velocity as a function of distances from the jet outlet.

Figure 19 shows the evolution of the mean conditional azimuthal vorticity for several different axial po- 


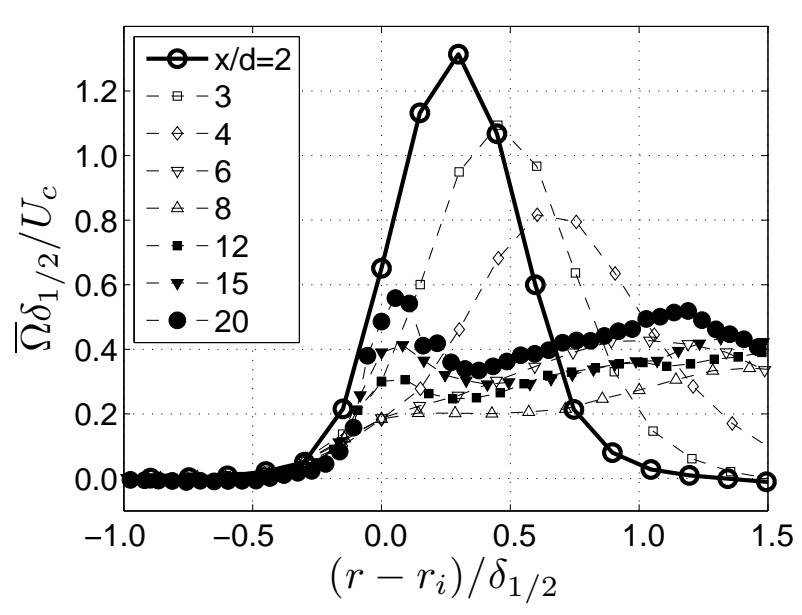

(a)

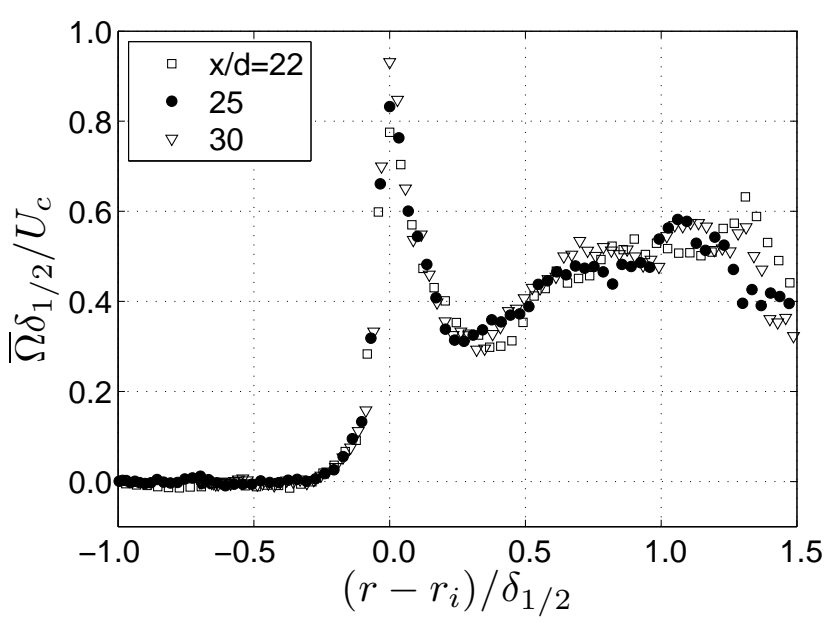

(b)

Fig. 19 Profiles of mean conditional azimuthal vorticity for several different distances from the jet exit, in the developing area (a) and the self-similar turbulent region (b) of the jet.

sitions with respect to the jet exit. The general pattern of the plots is the same as the profiles of $\bar{U}$. As mentioned, the existence of the peak in the graphs demonstrates the formation of vortex sheet structures at the interface location in the jet (Westerweel et al. [2009]). The results for the near field region $(x / d<8)$ shows that there is no peak in the profiles of $\overline{\Omega_{z}}$. The graphs show a gradual decay, followed by the emergence of a peak at the interface at $x / d \approx 8$. For $8<x / d<20$, the height of this peak continues to increase (figure 19a) until the normalized profile of $\overline{\Omega_{z}}$ evolves to a self-similar shape beyond $x / d=20$ (figure $19 \mathrm{~b}$ ).

A further examination of the evolution of the characteristics was carried out for mean conditional Reynolds shear stress for the locations mentioned previously and presented in figure 20. As mentioned, $\overline{u v}$ is not zero

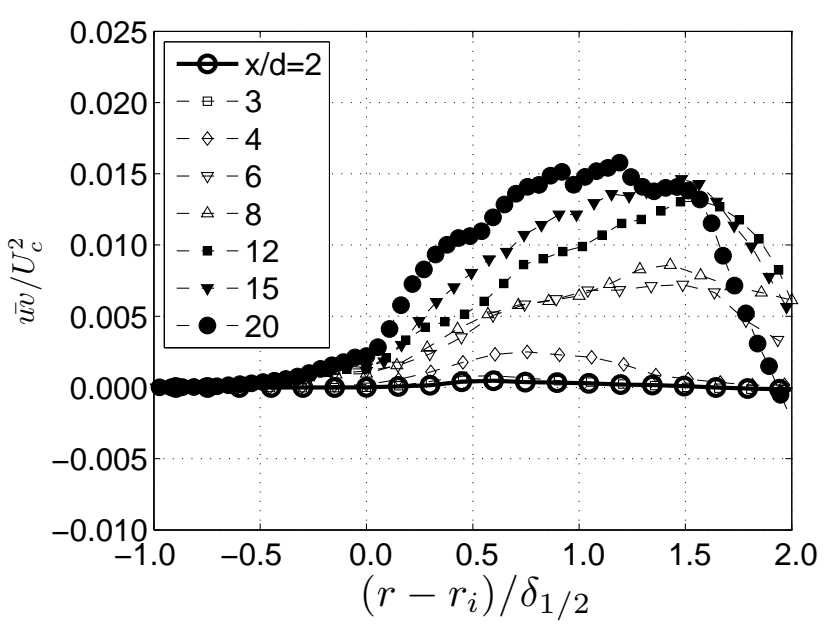

(a)

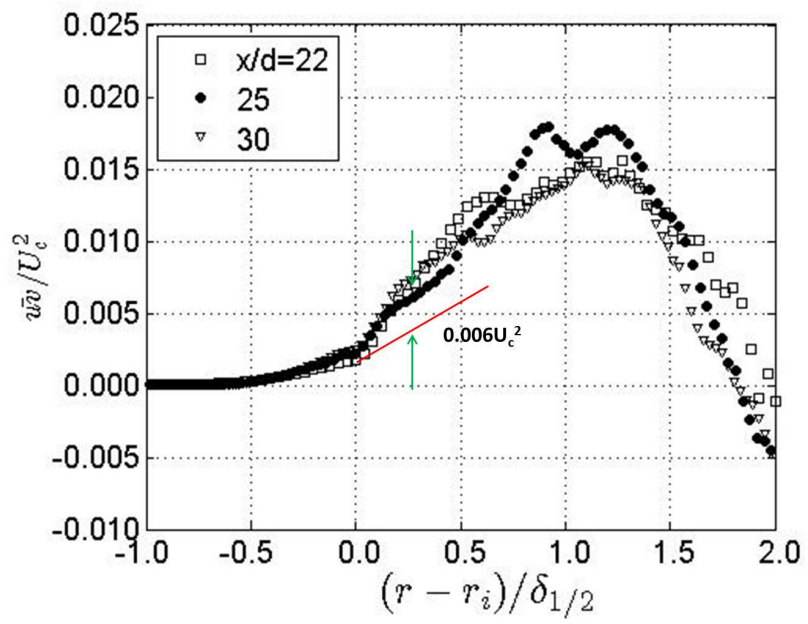

(b)

Fig. 20 Profiles of mean conditional Reynolds shear stresses for several different distances from the jet exit, in the developing area (a) and the self-similar turbulent region (b) of the jet.

at the interface and shows a jump like those in the $\bar{U}$ graphs, in the fully turbulent region. Figure 20a shows that $\overline{u v}$ for the near field area $(x / d<6)$ is zero. This value increases as the jet evolves, reaching a value of 0.0037 at the interface in the self-similar region. On the other hand, the evolution of the $\overline{u v}$ shows a pattern similar to the mean conditional axial velocity profiles. Furthermore, the value of $\Delta \overline{u v}$ at the interface for the self-similar region is estimated at $0.006 U_{c}^{2}$, which can be used in equation 3 . Using the values of $\Delta U$ and $\Delta \overline{u v}$, the propagation velocity of the interface $\left(E_{b}\right)$ was measured and found to be $0.066 U_{c}$, which is comparable with the results of the experiments of Westerweel et al. [2009] $\left(E_{b}=0.07 U_{c}\right)$. 


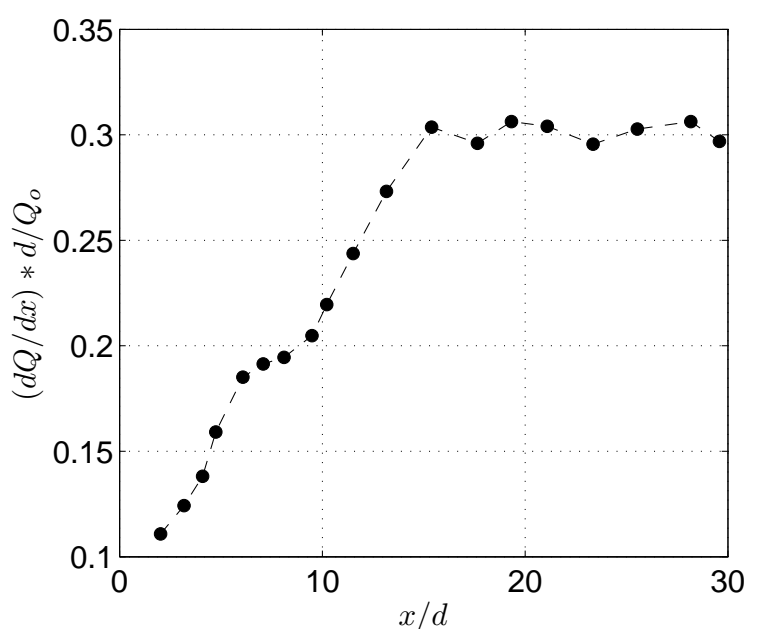

Fig. 21 The normalized entrainment rate versus downstream distance.

\subsection{Evolution of the Rate of Entrainment}

The PIV results in the developing region of the jet showed the creation and growth of a jump in the mean axial and Reynolds shear stress profiles. The side views of the jet indicated that the interface location in the transient region of the jet moved out into the quiescent ambient fluid with a non-linear profile, followed by a linear pattern in the fully turbulent region. It is evident that this interface propagation speed can affect the rate of entrainment:

$\frac{d Q}{d x}=\int\left(V_{i}-E_{b}\right) r d \theta$

where $V_{i}$ is the radial velocity at the interface. Figure 21 shows the rate of entrainment for different distances from the jet exit. It is clear that at the fully turbulent region the rate of entrainment reaches a constant value. Another observation of figure 21 is the considerable changes in the rate of entrainment at around $x / d=8$, which can support our earlier statement for the creation of the jump in the mean axial velocity profile. On the other hand, we can assume that the value of $E_{b}$ for $x / d<8$ is approximately equal to zero and increases between $x / d=8$ and $x / d=15$. The plot also shows that the self-similarity stage starts at about $x / d=15$.

\section{Conclusion}

In this paper, experimental data of high-resolution 2D$2 \mathrm{C}$ planar PIV has been presented for a turbulent round jet at Reynolds number in the range 3000 to 6500 . The main aim of this paper was to study physical and statistical properties of the flow near the boundary between turbulent and non-turbulent flow regions, with a particular emphasis on the evolution of conditionally averaged properties from the laminar to the fully turbulent region.

Consistent with published data in the open literature (see Westerweel et al. [2005, 2009] and Anand and Boersma [2009]), profiles of mean conditional axial velocity show a clear jump at the interface in the selfsimilar region. This jump in $\bar{U}$ does not exist in the near field of the jet $(x / d<8)$. The magnitude of the jump increases linearly from zero when $x / d \approx 8$ to $0.09 U_{c}$ in the self-similar region. This same pattern is seen for the profiles of the mean conditional Reynolds shear stress. The value of the jump for the latter property $\overline{u v}$ started to increase from its zero values at $x / d=6$, approaching to its maxima at about $x / d=20$. The evolution of the mean conditional azimuthal vorticity profiles shows that there is no peak in the profiles at the interface for $x / d<8$ (and is in agreement with the results of $\bar{U}$ ), at which the peak emerges and evolves in the transitional region reaching self-similar values at $x / d=20$.

Finally, the profile of rate of entrainment in the developing region agrees with our earlier conclusion regarding the emergence of the jump and the location of the start of the self-similar region.

Acknowledgements The authors wish to gratefully thank the financial support of the Australian Research Council (ARC).

\section{References}

R. K. Anand and B. J. Boersma. Detection of turbulent/non-turbulent interface for an axisymmetric turbulent jet: evaluation of known criteria and proposal of a new criterion. Exp. Fluids, 47:995-1007, 2009.

D. K. Bisset, J. C. R. Hunt, and M. M. Rogers. The turbulent/non-turbulent interface bounding a far wake. J. Fluid. Mech., 451:383-410, 2002.

G. L. Brown and A. Roshko. On density effects and large structure in turbulent mixing layers. J. Fluid. Mech., 64:775-816, 1974.

S. Corrsin and A. L. Kistler. The free-stream boundaries of turbulent flows. NACA TN-3133, TR-1244, pages 1033-1064, 1954.

B. C. Da-Silva and J. C. F. Pereira. Invariants of the velocity-gradient, rate-of-strain, and rate-of-rotation tensors across the turbulent/ nonturbulent interface in jets. Phys. Fluids, 20:055101,1-18, 2008.

C. B. da Silva and R. R. Taveira. The thickness of the turbulent/nonturbulent interface is equal to the radius of the large vorticity structures near the edge 
of the shear layer. Phys. Fluids, 22:121702, 1-22, 2010 .

W. J. A. Dahm and P. E. Dimotakis. Measurements of entrainment and mixing in turbulent jets. AIAA J, 25:1216-1223, 1987.

P. E. Dimotakis. The mixing transition in turbulent flows. J. Fluid. Mech., 409:69-98, 2000.

J. A. Ferre, J. C. Mumford, A. M. Savill, and F. Giralt. Three-dimensional large-eddy motions and fine-scale activity in a plane turbulent wake. J. Fluid. Mech., 210:371-414, 1990.

J. O. Hinze. Turbulence, 2nd edition. McGraw-Hill, 1975.

M. Holzner and B. Luthi. Laminar superlayer at the turbulence boundary. Phys. Rev. Lett., 106:134503, 2011.

M. Holzner, A. Liberzon, M. Gaula, and A. Tsinober. Generalized detection of a turbulent front generated by an oscillating grid. Exp. Fluids, 41:711-719, 2006.

M. Holzner, A. Liberzon, N. Nikitin, W. Kinzelbach, and A. Tsinober. Small-scale aspects of flows in proximity of the turbulent/nonturbulent interface. Phys. Fluids, 19, 071702, 2007.

M. Holzner, A. Liberzon, N. Nikitin, B. Luhti, W. Kinzelbach, and A. Tsinober. A lagrangian investigation of the small-scale features of turbulent entrainment through particle tracking and direct numerical simulation. J. Fluid. Mech., 598:465-475, 2008.

J. C. R. Hunt, N. D. Sandham, J. C. Vassilicos, B. E. Launder, P. A. Monkewitz, and G. F. Hewitt. Developments in turbulence research: a review based on the 1999 programme of the isaac newton institute, cambridge. J. Fluid. Mech., 436:353-391, 2001.

J. C. R. Hunt, I. Eames, and J. Westerweel. Mechanics of inhomogeneous turbulence and interfacial layers. J. Fluid. Mech., 554:499-519, 2006.

H. J. Hussain. A selection of test cases for the validation of large-eddy simulations of turbulent flows. Technical report, 1998.

L. S. G. Kovasznay, V. Kibens, and R. F. Blackwelder. Large-scale motion in the intermittent region of a turbulent boundary layer. J. Fluid. Mech., 41:283-325, 1970.

J. Mathew and A. J. Basu. Some characteristics of entrainment at a cylindrical turbulence boundary. Phys. Fluids, 14:2065-2072, 2002.

M. G. Mungal, P. S. Karasso, and A. Lozano. The visible structure of turbulent jet diffusion flames: largescale organization and flame tip oscillation. Combust. Sci. Technol., 76:165-185, 1991.

N. R. Panchapakesan and J. L. Lumley. Turbulence measurements in axisymmetric jets of air and helium. part 1. air jet. J. Fluid. Mech., 246:197-223, 1993.

J. Philip and I. Marusic. Large-scale eddies and their role in entrainment in turbulent jets and wakes. Phys. Fluids, 24:055108, 2012.

S. B. Pope. Turbulent Flows. Cambrige University Press., 2000.

J. Soria. Multigrid approach to cross-correlation digital PIV and HPIV analysis. In Proceedings of 13th Australasian Fluid Mechanics Conference. Monash University, Melbourne, Australia, 1998.

J. Soria, J. Cater, and J. Kostas. High resolution multigrid cross-correlation digital PIV measurements of a turbulent starting jet using half frame image shift film recording. Opt. Las. Technol, 31:3-12, 1999.

A. Tsinober. An Informal Introduction to Turbulence. Kluwer, 2001.

K. von Ellenrieder, J. Kostas, and J. Soria. Measurements of a wall-bounded turbulent, separated flow using HPIV. Journal of Turbulence, 2:1-15, 2001.

T. H. Weisgraber and D. Leipman. Turbulent structure during transition to selfsimilarity in a round jet. Exp. Fluids, 24:210-224, 1998.

J. Westerweel, T. Hofmann, C. Fukushima, , and J. C. R. Hunt. The turbulent/non-turbulent interface at the outer boundary of a self-similar turbulent jet. Exp. Fluids, 33:873-878, 2002.

J. Westerweel, C. Fukushima, J. M. Pedersen, and J. C. R. Hunt. Mechanics of turbulent-non-turbulent interface of a jet. Phys. Rev. Lett, 95,174501, 2005.

J. Westerweel, C. Fukushima, J. M. Pedersen, and J. C. R. Hunt. Momentum and scalar transport at the turbulent/non-turbulent interface of a jet. J. Fluid. Mech., 631:199-230, 2009. 


\section{University Library}

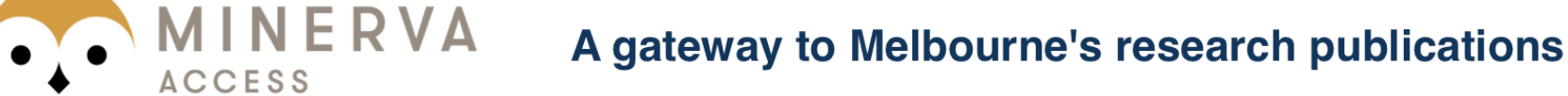

Minerva Access is the Institutional Repository of The University of Melbourne

Author/s:

Khashehchi, M;Ooi, A;Soria, J;Marusic, I

Title:

Evolution of the turbulent/non-turbulent interface of an axisymmetric turbulent jet

Date:

2013-01-01

Citation:

Khashehchi, M., Ooi, A., Soria, J. \& Marusic, I. (2013). Evolution of the turbulent/nonturbulent interface of an axisymmetric turbulent jet. EXPERIMENTS IN FLUIDS, 54 (1), https://doi.org/10.1007/s00348-012-1449-3.

Persistent Link:

http://hdl.handle.net/11343/283064 